\title{
An Alumni Portal and Tracking System
}

\author{
Baibhav Bista ${ }^{a}$, Aman Shakya ${ }^{b}$, Basanta Joshi ${ }^{c}$, Anusandhan Pokhrel, \\ Lumanti Dangol, Mohit Kedia, Daya Sagar Baral \\ Department of Electronics and Computer Engineering, Pulchowk Campus, Institute of Engineering, Tribhuvan University, Nepal \\ Corresponding Authors: ${ }^{a}$ baibhavbista@gmail.com, ${ }^{b}$ aman. shakya@ioe.edu.np, ${ }^{c}$ basanta@ioe.edu.np
}

Received: 2020-07-24

Revised: 2021-01-23

Accepted: 2021-02-19

\begin{abstract}
:
Alumni tracking is a difficult task for any institution that has been running for a long time. The Department of Electronics and Computer Engineering (DOECE), Pulchowk Campus, IOE, has been offering undergraduate programs since 1994 A.D. and masters programs since 2001 A.D. The existing information about the alumni of the department is unmanaged and out of date. This paper presents a web-based system to integrate data of alumni into a well-managed database, and to act as a portal where alumni can update their current status and view online alumni yearbooks. This work aims to make the task of alumni tracking easier and simpler for the department. The web-based portal has been accessed by many alumni of the department starting from the earliest batches to update their information and see the whereabouts of others. From the collected alumni responses, some preliminary insights observed were: (a) it was seen that alumni seem to be heterogeneous both in their job positions and employers, however, a large portion are employed in the software sector, (b) most alumni (60\%) are currently residing in Nepal, and (c) for further education, the USA (39\%) seems to be the favored choice, followed closely by Nepal (32.5\%).
\end{abstract}

Keywords: Alumnus, Web Portal, Alumni Tracking, DOECE

\section{Introduction}

Alumni associations play an essential role in higher education institutions, as they provide networking opportunities and contact between the university and the alumni or among the alumni. However, the lack of communication between the alumnus, faculty and the department as a whole leading to the lack of updated data of alumni hinders the effective development of an alumni association. The lack of data update can be tied to disinterest or ignorance of the alumnus about the potential of an alumni association to promote a professional network, academic and even personal relationships. Similarly, the need for an effective system to maintain these records and to update them frequently becomes increasingly pressing as the number of students enrolled in the campus continues to increase. One of the ways to consolidate the relationship between alumni and educational institutions is to create a virtual and interactive portal to provide useful information to the alumni to help extend the contact between the alumni and the university to beyond the period of course completion, regardless of geographic location or time.

The main research question that the paper attempts to address is - how to collect and maintain records of alumni with minimal requirement of centralized data collection and manual entry. Enticing and motivating alumni, who have already graduated and busy in their own ways, to enter their latest profile is also challenging.

As a partial solution to the above problem, the main research contribution of this work is the development and implementation of an online system with the following objectives:

- To keep the records of all alumni of the Department of Electronics and Computer Engineering (DOECE), Pulchowk Campus including the current academic and/or employment status, their whereabouts.

- To publish an online alumni yearbook as a stepping stone for inter-alumni and alumni-student 
communication.

- To automate the process of alumni tracking by allowing alumni to update and maintain their own information ensuring an up-to-date alumni database.

The prospect of having one's latest profile listed in the year book, along with his/her batch mates would motivate the alumni to some extent to update their information online. Moreover, initial data of the alumni can be automatically fetched from the campus database of students, avoiding cumbersome manual data collection and entry.

From this paper, similar system builders and researchers may get an idea of the main problems one encounters when dealing with digitization and integration of student records. The techniques and heuristics for dealing with inconsistent and duplicate records may also prove applicable to other domains.

\section{Related Works}

Alumni portals act as common platform to serve the cause of integrating all the stakeholders of educational institutes such as alumni, college students and faculties to avail the guidance and knowledge sharing on various domains in many colleges and universities. Mijic, Jankovic [1] discuss a possible solution to information system for tracking information about alumni, and its possible contribution to improvement and innovation of study programs. Bakioğlu et al. [2] held a survey among 142 volunteers of all graduates of Marmara University Atatürk Education Faculty. The data acquired from the survey indicated that the graduates needed the tracking system, career planning center and alumni association and were positive to be approached via web and pointed out that former graduates providing employment opportunities to the newly graduates as well as sharing job experiences and career guidance will bring up more efficient results. Some alumni portals and organizations, both outside and inside Nepal are discussed in this section.

Harvard University Alumni portal [3] allows alumni to login to connect with other alumni or students, along with search functionality. The portal also provides information about recent and upcoming events and programs, such as but not limited to networking events, career webinars. Alumni can get to know about the clubs and shared interest groups. The alumni portal also acts as a portal for volunteer fundraising for the university. The alumni portal of IIT Kanpur Alumni Association [4] has an alumnus mentoring and buddy system which pairs alumni and students, with the alumni mentoring the students and helping them meet their career goals. The portal also provides access to the number of alumni charters and city ambassadors. The portal also has a dedicated career opportunities section where jobs can be listed and applied from.

Society of Ex-Budhanilkantha Students (SEBS) was incepted in the year 1982 as an alumni association of Budhanilkantha School. SEBS has an online portal [5], with information about the school, about recent and upcoming news, and announcements. It also acts as platforms for different donation programs. The portal provides access to different charters of SEBS like SEBS-North America, SEBS-UK. The portal also has discussion forums with options like general forums, batch message board, archive and advanced search and resources and links for the students to help with their education, chat room and feedback options.

Institute of Engineering Alumni Association (IOEAA) was initially established in 2009. IOE and IOEAA have agreed to work together in academic as well as networking of its graduates. Campus alumni association is currently collecting information from the alumni members through web forms. It is working on initiating to publish the annual report of the Campus and link the Education Management Information System (EMIS) and alumni portal with the public domain (Website).

\section{Methodology}

\subsection{Overview}

The most important part of every alumni system is the alumni data, and so we focus on the phases of data flow. After collection from a number of sources, alumni records went through preliminary cleaning and integration. Then, possibly duplicate records (for alumni who enrolled in more than one program in the department) were flagged using some heuristics and merged. The last phase of the methodology is an ongoing process of alumni updating their information through the deployed system. Details of each phase are described in the following sub-sections. 


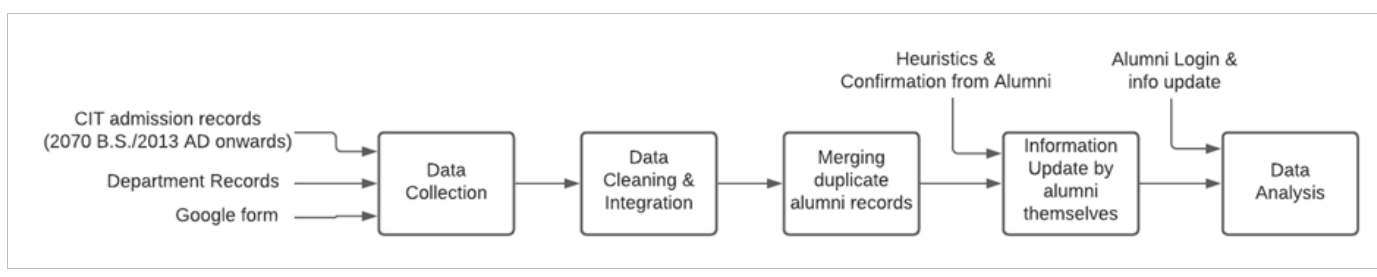

Figure 1: Overview of Methodology

\subsection{Data Collection}

Data was collected from the following sources:

- From the admission data recorded by Center of Information Technology, Institute of Engineering, data of bachelors' students (BCT and BEX/BEI) from 2070 B.S. to 2075 B.S. (2013 A.D. to 2018 A.D.) was obtained.

- Previous alumni records were obtained from the department records: BCT from 2055 B.S. (1998 A.D.), BEX from 2051 B.S. (1994 A.D.), MScCSKE from 2069 B.S. (2012 A.D.) and MScICE from 2058 B.S. (2001 A.D.). These records were inconsistent, and had to undergo cleaning before they could be input into the database. Photos were collected by a team of student volunteers by manually scanning passport-sized photos from paper records and imported separately.

- While the system was being built, a google form was created to collect responses about current status of the alumni. The form collected data about location, employment status, areas of expertise, further education, alongside identifying information such as roll number, enrolled and passed year and program. This form is now closed, integrated with the database, and alumni can now update their information through this system itself.

\subsection{Data Cleaning and Integration}

The main hurdle we found was integrating the alumni data from various sources, since they were of varying states and formats. First of all, a schema was developed taking into account the various fields of data we had. Data obtained from various sources were then cleaned, and converted into the new schema, and merged. Tools such as pandas (Python library), psql (PostgresSQL command-line utility), MS Excel were used for this purpose. Some problems observed were:

- Data did not have a consistent format, even those from the same source. Taking example of Date of Birth (DOB) for the date 2nd January 1990 (or equivalently 2046 Poush 18), they were in different formats like '1990/01/02', '02-01-90', '2049-09-18', '18/09/049', etc. For some sources, the process of converting to a standard date format (we chose B.S. with format YYYY/MM/DD) could be scripted, while for some, they had to be converted to a common format painstakingly by hand before conversion to our standard date format.

- The program codes had been changed a number of times, before settling onto a standardized 3-character or 5-character format (Bachelors and Masters programs respectively), that uniquely identifies any program across all the different departments of the Institute of Engineering (IOE). This had to be taken into account and all previous alumni were also assigned this program format, for simplicity and consistency.

\subsection{Merging Duplicate Alumni Profiles}

Another challenge was the duplicate profiles of alumni who had studied two or more programs offered by the department e.g. an alumnus who studied both Bachelors and Masters in the department. As far as possible, these duplicate records were combined. Some heuristics were used to identify possible duplicate profiles, which were then merged after confirmation by the alumni themselves. Some heuristics used to identify possible duplicates were:

i) Having the same (or similar) names. For example, middle names were sometimes omitted in the records so two candidate profiles would be flagged as possible duplicates if their first and last names matched, even if the middle name didn't.

ii) Having same (or similar) father and mother names.

iii) Having the same phone number

iv) Having same email address 
v) Having some of the heuristics above and the same date of birth

vi) Candidate profiles with enrollment in different programs offered by the department during overlapping time periods were not flagged as possible duplicates, even if they satisfied heuristics above.

\subsection{Information update by alumni themselves}

After the system was up and running, alumni could and can still login into the system and update their information themselves. Some insights that we obtained from this have been illustrated in the Results section of this paper. This is the last and the continually running part of the methodology. Alumni can view their and their batch mates' profiles in an online yearbook, which also provides incentive to update their profile.

\section{Requirements}

\subsection{Use Case Diagram}

The system is designed for use by three categories of users: Public, Alumni and Admins. Public users can view yearbooks. Alumni users can login and update their information in addition to being able to view yearbooks. Admin users have the most privileges and have access to admin dashboard with features like advanced search and functionality like import and export data.

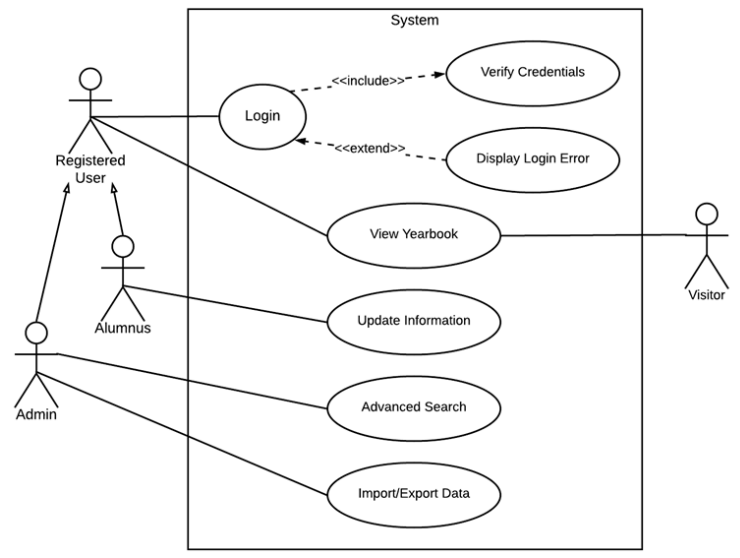

Figure 2: Use Case diagram for the system

\section{Implementation}

\subsection{Architecture of the proposed system}

Web-based Frontend (Client side):

The system has a publicly hosted frontend user interface (http://manaslu.pcampus.edu.np/alumni/) where people can view yearbooks of different programs and years. In addition to that, this frontend enables alumni to login into the system and update their information. Admins can access a separate admin portal, which has advanced searching, filtering functionality as well as features for importing and exporting data.

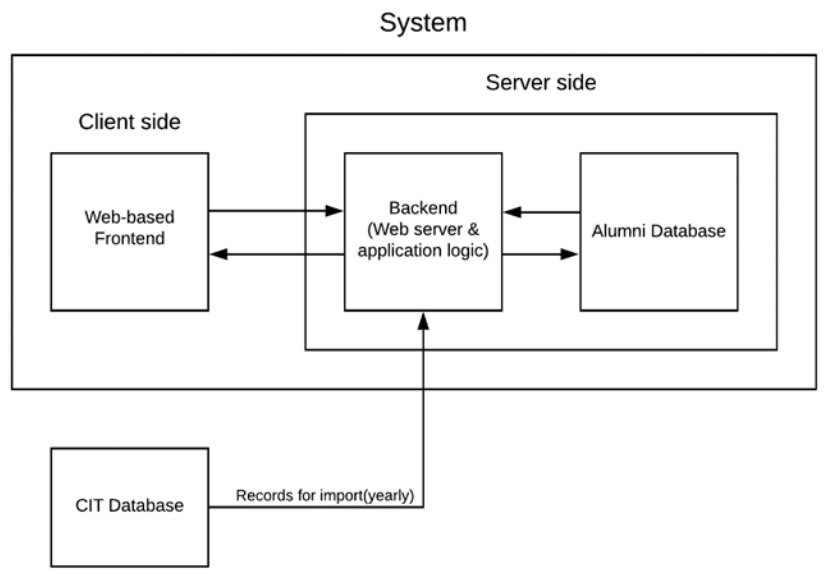

Figure 3: Block diagram of System Architecture

\section{Server side:}

The system is hosted on DoECE server and consists of following two parts:

- Backend: The backend handles the requests from clients which come through the web-based frontend, bridging the gap between client actions (e.g. viewing yearbook, updating info) and the database. It also handles all application logic, including data imports from CIT.

- Alumni Database: This is the store of all records. The database design and implementation are described in detail in sections 5.2 and 5.3 respectively.

\section{Center of Information Technology (CIT) Student Database:}

CIT has been managing electronic admission records for Pulchowk campus since 2070 B.S. (2013 A.D.). As a result, CIT is a recurring source of authentic student records, which form the basis of alumni records for this system. Data from CIT records is imported into our system on a yearly basis using a Python script after some manual pre-processing. 


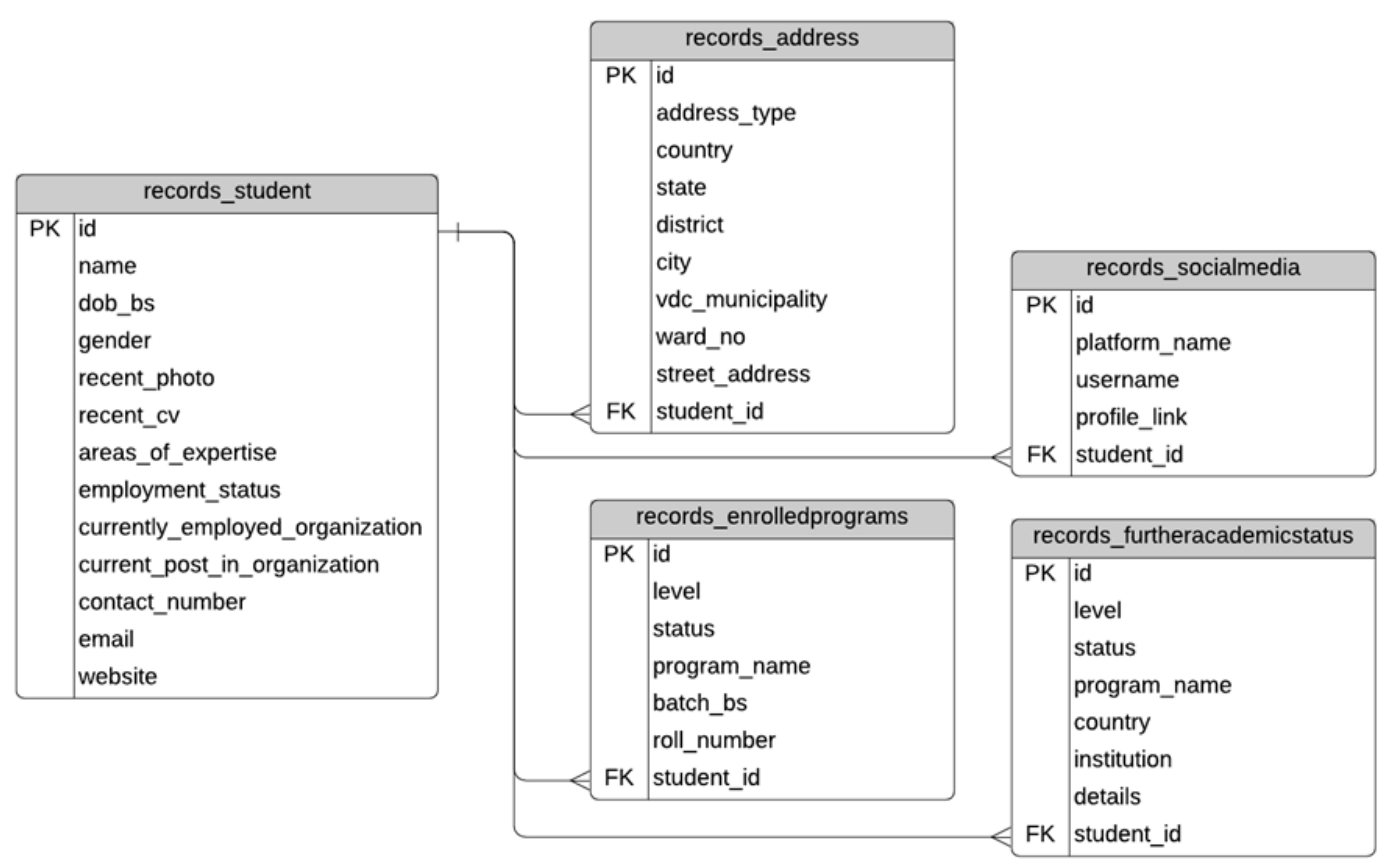

Figure 4: Schema diagram of the database

\subsection{Conceptual Database Design}

The database has a simple design, with a main 'records_student' table and separate tables for attributes which may have multiple instances - address, social media, enrolled programs (in DOECE) and further academics, stored in tables 'records_addresses', 'records_socialmedia', 'records_enrolledprograms' and 'records_furtheracademicstatus' respectively. The simplified schema diagram presented in Figure 4 also provides important details about types of information collected from alumni.

\subsection{Technical Details of implementation}

The system was built using Django, a Python web framework, and is served using Gunicorn (application server) and Nginx (webserver). The database used is PostgreSQL 9.6.15. The system is hosted on a CentOS 7 server. Django and Python were chosen due to authors' familiarity as well as due to its security, reliability and flexible ORM. PostgreSQL was chosen for its reliability, security and performance.

\section{Results and Analysis}

\subsection{Screenshots of the System}
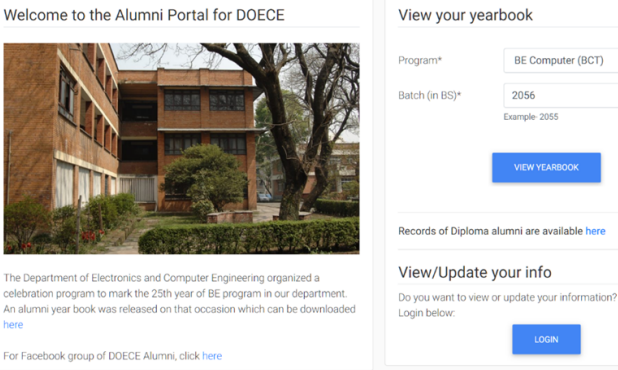

Figure 5: Screenshot of Home Screen

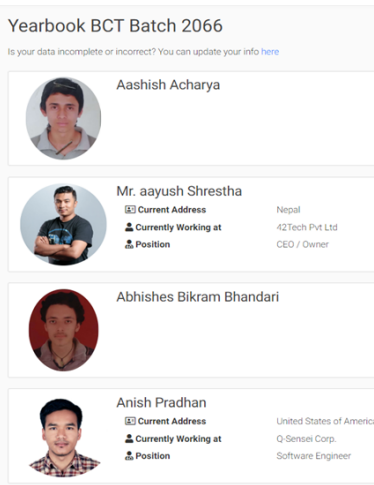

Figure 6: Screenshot of Sample Yearbook 


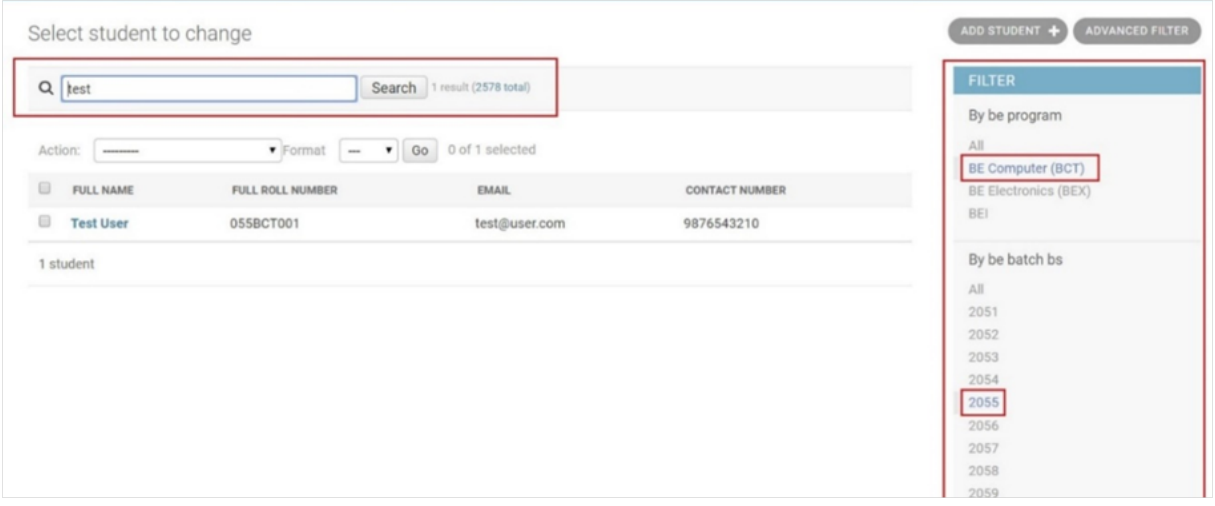

Figure 7: Admin Panel Screenshot: Faceted searching, filtering using common attributes like program name, batch (here: search phrase $=$ 'test', program name $=$ BCT, batch $=2055$ )

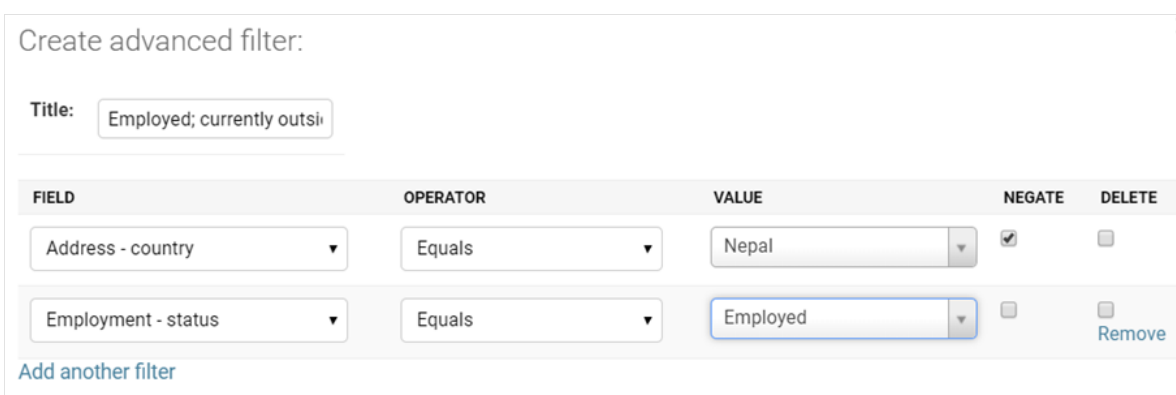

Figure 8: Admin Panel - Custom filters (here: a filter for alumni that are employed and currently living outside Nepal)

\subsection{Analysis of collected data}

A total of 2155 entries were collected till 06/06/2020, with 643 alumni filling up the detailed forms regarding current location, academic \& employment status. In this section, preliminary observations and results from these 643 responses are described.

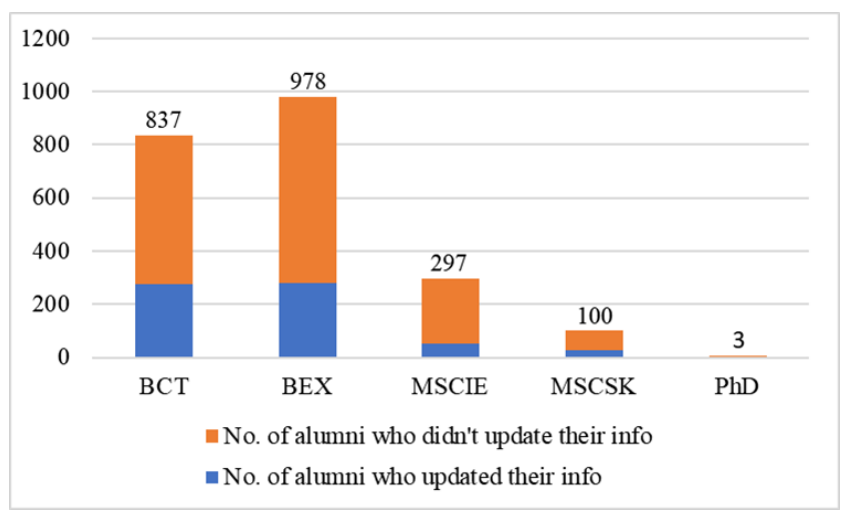

Figure 9: Bar Diagram for the number of alumni who updated their information with the total number of alumni for each program

The chart in Figure 9 shows the total number of alumni of each program till date and the number of alumni who updated and didn't update their information, grouped on the basis of the program they studied in the department.

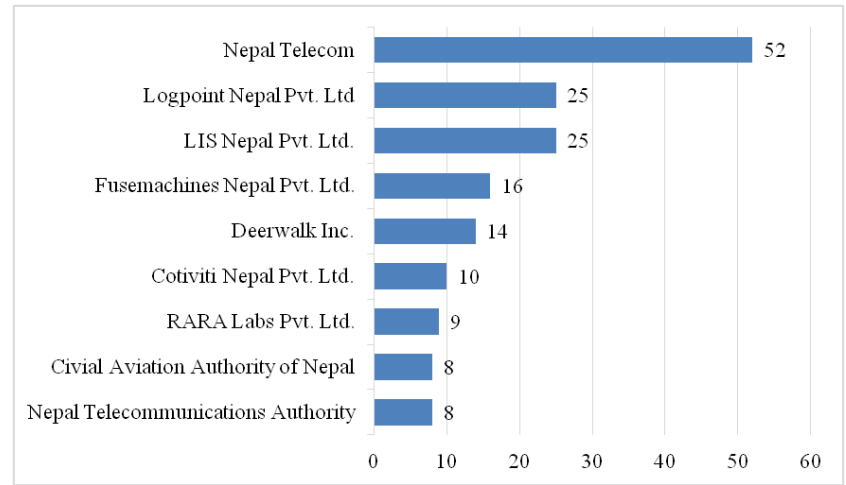

Figure 10: Bar Diagram for Alumni's place of employment (includes organizations with more than 5 alumni)

The chart in Figure 10 shows current place of employment of alumni. It was observed that the highest number of alumni are employed currently in Nepal Telecom with 52 alumni. Only the organizations with 5 or more alumni employees were included in the figure. 
Four hundred and fifty six alumni were from organizations that did not fit this criteria i.e. with less than 5 employees who were DOECE Alumni. This indicates that the companies where alumni are currently working is highly heterogeneous and the concentration of alumni in a single company is less prominent.

Current job positions of alumni are charted in figure 11. It was observed that four out of top five current job positions of alumni are related to the field of Software Engineering. Similar to figure 10, this figure also only includes job positions with more than 5 alumni. Three hundred and forty alumni had job positions which did not fit this criteria which signifies the heterogeneity of the positions. However, the positions seem to be highly concentrated towards Software Engineering \& Development. The reason for alumni being employed in software much more than hardware industry may be due to the comparatively smaller market for hardwarerelated jobs in Nepal.

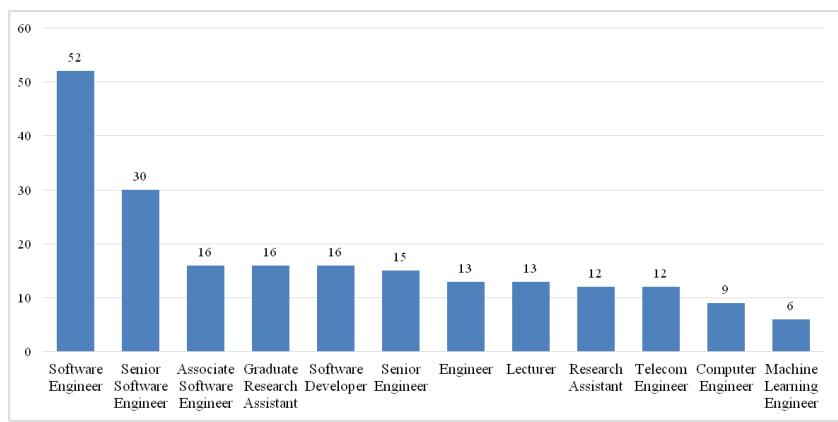

Figure 11: Bar Diagram of current job position (only includes positions with more than 5 alumni)

From figure 12 (chart of current residence of alumni), it was observed that a majority of alumni are currently residing in Nepal itself. Alumni who are living abroad are mostly concentrated in USA and then in Australia.

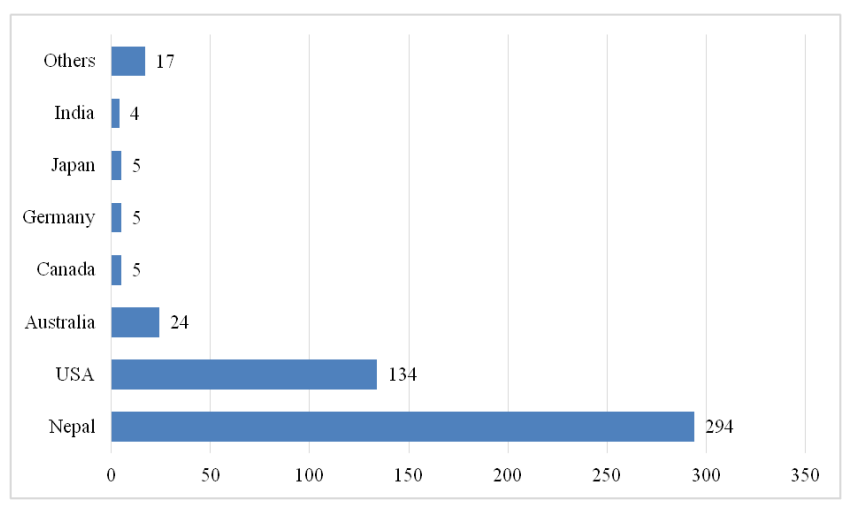

Figure 12: Bar Diagram of current residence of Alumni
Table 1 lists the number of alumni who have gone for further education, both ongoing and completed, grouped by level (Masters, PhD, Post Doc.).

Table 1: Alumni who are undergoing or have completed further education, grouped by Level

\begin{tabular}{|l|c|c|c|}
\hline \multirow{2}{*}{ Higher Studies Level } & \multicolumn{3}{|c|}{ No. of Graduates } \\
\cline { 2 - 4 } & Ongoing & Completed & Total \\
\hline Masters & 68 & 167 & 235 \\
\hline Ph.D & 21 & 23 & 44 \\
\hline Post Doc. & 0 & 3 & 3 \\
\hline
\end{tabular}

Out of these records, 262 have data about country of study, which is illustrated in Fig. 13. It can be seen that a majority of alumni prefer USA for abroad studies while the number of alumni choosing Nepal for further studies is also substantially high. It also shows that the countries preferred by alumni for further students are limited in number.

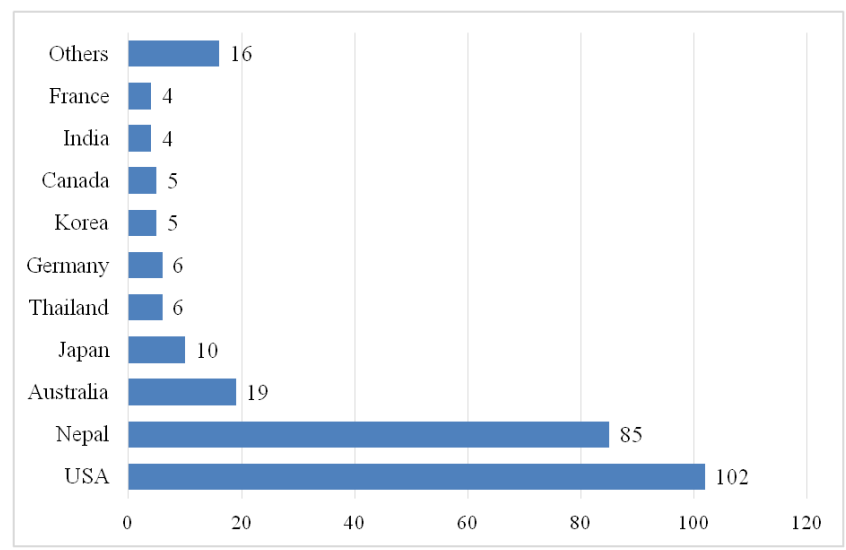

Figure 13: Bar Diagram of further studies of Alumni
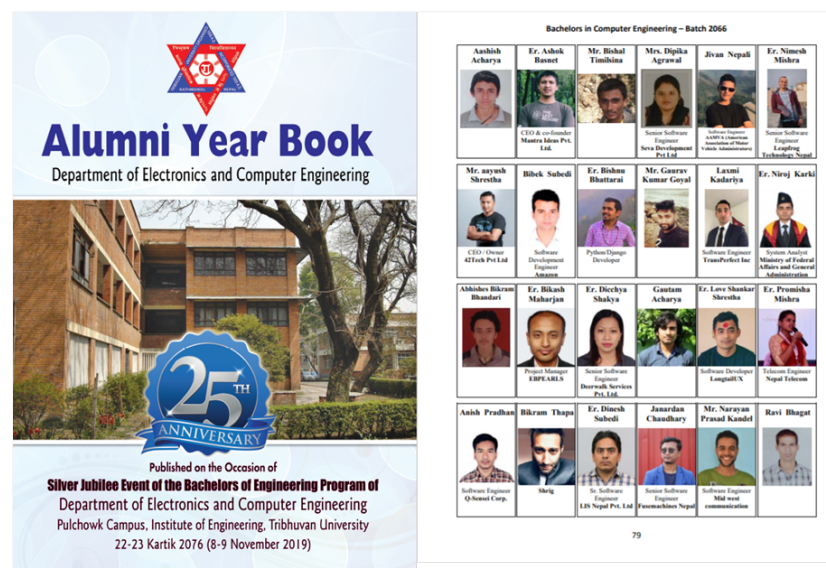

Figure 14: Front cover along with an inside page of Alumni Year Book

The Department of Electronics and Computer 
Engineering organized a celebration program to mark the 25th year of BE program in the department on 9th November, 2019. On that occasion, an alumni yearbook of the department was released, with pictures and current employment information of alumni. This yearbook was generated from this alumni database.

\section{Conclusions and Future Work}

In this paper, a web-based system is presented for collection and visualization of information of alumni of Department of Electronics and Computer Engineering, IOE, Pulchowk Campus. A new database is designed to integrate latest information from college administration in order to avoid variation in structure for current students.

From the data collected from 24/06/2019 up to $06 / 06 / 2020$, we can draw the following major conclusions:

- Companies where alumni work seem to be highly varied, with the largest portion in Nepal Telecom with 44 responses

- A significant portion of alumni currently work in the software industry.

- USA seems to be the most favored place for further education. This is also probably a cause for why USA seems to be the country with 2 nd highest number of alumni currently living, after Nepal.

Some recommendations for the future are:

- Due to lack of personally identifiable information like email addresses of past alumni, we had to authenticate using date of birth only. After sufficient emails have been collected through the update form (where email is a compulsory question), the authentication can be shifted to a more secure format like email-password pair.

- When majority of alumni have filled up the form, a comprehensive study can be done, building upon the preliminary survey done in this work.

- The system can be extended to be a hub for inter-alumni, alumni-student, and alumni-college communication for e.g. a place to host job vacancies, a place to organize and be informed of college events.

- Automated tracing can be carried out using LinkedIn and other platforms to update alumni information.

- The system can be implemented campus-wide, so as to have a central repository of all alumni of Pulchowk Campus.

\section{References}

[1] D. Mijic and D. Jankovic, "Towards Improvement of the Study Programme Quality: Alumni Tracking Information System," in ICT Innovations 2011, L. Kocarev, Ed. Berlin, Heidelberg: Springer Berlin Heidelberg, 2012, pp. 291-300.

[2] A. Bakioğlu, M. Yüksel, B. Akdağ, and A. N. Canel, "Alumni tracking system in higher education: a survey on alumni of Marmara University Atatürk Education Faculty," Yükseköğretim Dergisi, vol. 1, no. 2, pp. 65-79, 2011.

[3] "Harvard Alumni," [online]. Available: http://www.alumni.harvard.edu (Accessed 2020)

[4] "IIT Kanpur Alumni," [online]. Available: http://www.iitkalumni.org (Accessed 2020)

[5] "SEBS Online,"[online]. Available: http://www.sebsonline.org (Accessed 2020) 